\title{
LES POLITIQUES ET LES MÉDIAS EN GRANDE-BRETAGNE
}

\author{
David Deacon et Dominic Wring ${ }^{1}$
}

Même si l'été 2003 restera probablement longtemps gravé dans les mémoires pour les questions délicates soulevées par les journalistes et d'autres personnes quant à la probité du gouvernement britannique dans sa justification du bien-fondé de la guerre en Irak, les éditorialistes et les directeurs de rédaction ont également fait l'objet d'une surveillance minutieuse mais, dans leur cas, en ce qui concerne l'intrusion des médias dans la vie privée. Le rapport du comité spécial Culture, Médias et Sport de la Chambre des communes, publié en juin, a rouvert un dossier qui avait été officiellement traité pour la dernière fois voici dix ans, lors de la publication du rapport final du Comité Calcutt, qui mettait fin à cinq années de délibérations sur les critères des médias en cette matière. Au cours du processus de révision précédent, David Mellor, alors ministre du Patrimoine et ex-secrétaire d'État au Patrimoine, a tiré le signal d'alarme de façon mémorable en déclarant que les médias britanniques, en particulier les tabloïds populaires, se trouvaient « dans le train de la dernière chance ». Ironie du sort, le ministre n'est pas resté en fonction assez longtemps pour juger s'il aurait été nécessaire de réagir à cette menace. En effet, il a été poussé à la démission quelques mois avant la publication du rapport final Calcutt, après avoir été égratigné par une série de révélations parues dans la presse sur son empressement à

1 Senior Lecturers in Communication and Media Studies, Loughborough University.

Recherches en communication, $\mathrm{n}^{\circ} 24$ (2005). 
accepter des vacances offertes par des amis fortunés et sur ses frasques sexuelles avec une jeune actrice. L'arroseur arrosé, en somme.

En 2003, comme en 1993, le Comité de vigilance pour le respect de la vie privée a formulé des recommandations fermes en faveur de l'adoption de contrôles dans ce domaine mais, une fois encore, il semble hautement improbable que le gouvernement édicte une loi sur le respect de la vie privée dans un avenir proche. Quoi qu'il en soit, le fait que la classe politique soit revenue sur le sujet aussi rapidement et de manière aussi approfondie, démontre le caractère durable de la préoccupation publique en la matière et la conviction répandue que l'intrusion des médias dans la vie privée des citoyens et des personnalités publiques est trop souvent motivée par un voyeurisme et un érotisme gratuits plutôt que par une défense sérieuse de l'intérêt public.

Cet article examine un aspect du débat: dans quelle mesure les élus du Royaume-Uni sont soumis à l'intrusion des médias dans leur vie personnelle. Ce domaine est le plus controversé de l'ensemble du débat sur le droit de l'individu à une vie privée. L'article 8 de la Convention européenne des droits de l'homme dit: «Toute personne a droit au respect de sa vie privée et familiale, de son domicile et de sa correspondance », mais il est largement admis que ce droit n'est pas absolu. Comme le Comité spécial l'a fait observer : «Il est des situations limitées où il peut et doit être violé, lorsque le crime, la corruption ou l'hypocrisie sont cachés sous le couvert de la confidentialité et qu'il ne fait aucun doute que la vérité doit être révélée au grand jour dans l'intérêt public » (p. 9). L'élection (ou la désignation) à des fonctions publiques confère à la fois du pouvoir et des responsabilités, et il est indéniable que plus un politique ou une personne nommée à une charge publique a de l'influence, plus la défense de l'intérêt public est susceptible d'être invoquée pour justifier toute intrusion dans ses activités privées et ses motivations intimes. La question de la mesure dans laquelle les personnages publics transigent sur leur droit à une vie privée est encore compliquée par d'importantes tendances au "promotionalisme » politique au Royaume-Uni, où la projection des qualités personnelles des politiques est devenue un ingrédient encore plus significatif dans le marketing mix. Une ligne intéressante du récent rapport du Comité spécial semble indiquer qu'il est possible de distinguer « les individus qui ont des responsabilités publiques $\underline{o u}$ ceux qui ont recherché la publicité pour arriver à leurs fins » (l'italique a été ajouté). Cette distinction semble presque impossible à appliquer dans l'analyse de la scène politique britannique contemporaine. 


\section{Politiques et vie privée}

Il est manifeste que l'effacement des frontières entre la vie privée et la vie publique des personnalités politiques a atteint un degré qui dépasse largement celui des autres pays. La vie privée des politiques est considérée, plus qu' ailleurs, comme un terrain de chasse idéal. Les révélations d'un tabloïd britannique sur des problèmes de couple du Chancelier allemand Gerhard Schröder, alors que cette affaire avait été ignorée par les médias allemands, en sont une parfaite illustration. M. Schröder a immédiatement déposé une demande d'injonction temporaire auprès d'un tribunal de Hambourg interdisant le Mail on Sunday de publier toute autre information sur le sujet. En réponse, le journal a fait paraître un nouvel article de deux pages et un éditorial intitulé « Désolé, Herr Schröder mais vous ne dirigez pas la Grande-Bretagne... du moins pas encore ». Le Chancelier allemand y était décrit comme « un politique vaniteux... qui ne peut supporter la critique ». Ces articles étaient accompagnés d'un « message à nos lecteurs allemands », rédigé dans la langue de Goethe, les invitant à appeler un numéro de téléphone gratuit s'ils connaissaient d'autres révélations que M. Schröder pourrait trouver embarrassantes « et dont la presse allemande devrait parler».

Cette culture journalistique plus indiscrète possède certaines caractéristiques collatérales. Au Royaume-Uni, aucun politique n'est une île. La famille, les amis et les collaborateurs sont également considérés comme des cibles légitimes de l'attention des médias, uniquement en raison de leurs liens personnels avec un élu. Pour citer deux exemples récents liés au Premier ministre britannique, à la fin 2001, plusieurs journaux ont cherché a pousser Tony Blair à déclarer si son bébé avait reçu le vaccin controversé contre la rougeole, les oreillons et la rubéole, arguant que cette information était d'intérêt public puisque le gouvernement insistait sur la sécurité de ce triple vaccin malgré ses liens avec des troubles intestinaux et l'autisme. À cette occasion, M. Blair a résisté à la pression et n'a pas communiqué cette information personnelle en prétextant qu'il n'était pas en mesure de fournir à tout moment un « commentaire actualisé » sur la santé de son fils. En revanche, le Premier ministre et son épouse ne sont pas aussi bien parvenus à contrer les spéculations des médias à propos de l'achat par Cherie Blair d'un appartement pour son fils. L'intérêt des médias a été déclenché par la participation dans la transaction de Peter Foster, un escroc condamné et frappé d'un mandat d'expulsion qui sortait alors avec une amie proche de Mme Blair, qui était également sa conseillère personnelle en mode 
de vie. De nombreuses voix se sont élevées pour indiquer que Mme Blair, en tant qu'avocate expérimentée, avait prodigué à M. Foster des conseils au sujet de son expulsion. Le service de presse du Premier ministre a initialement récusé ces affirmations, avant de se rétracter partiellement lorsque les spéculations médiatiques se sont intensifiées et que de nouveaux faits ont été révélés. Ce scandale, connu sous le nom de « Cheriegate », s'est poursuivi pendant plusieurs semaines et ne s'est apaisé que lorsque Cherie Blair est apparue éplorée en public pour faire une déclaration visant à démentir tout mauvais comportement. Le fait que les éminences grises de la communication gouvernementale aient agi si rapidement pour réfuter l'affaire, démontre combien ces révélations étaient considérées comme une menace importante pour la crédibilité du Premier ministre et de son gouvernement.

Cette espèce de curiosité intense à propos des affaires privées des personnalités politiques et de leurs collaborateurs est un phénomène relativement récent. En réalité, entre le début et la moitié du $\mathrm{XX}^{\mathrm{e}}$ siècle, les journalistes britanniques étaient réputés pour leur discrétion dans leurs rapports avec les élites politiques. Vous ne trouverez, dans les archives des médias, aucune trace d'une quelconque révélation contemporaine sur le penchant de Churchill pour l'alcool ou sur la maitresse de Lloyd George. Bien entendu, la fascination actuelle pour la vie privée des grandes figures politiques ainsi que de leurs amis et leur famille (et il y a une corrélation entre l'importance d'un politique et le degré d'intérêt des journalistes pour sa vie privée) ne se manifeste pas uniformément dans tous les secteurs des médias britanniques. Il va sans dire que les tabloïds populistes à grand tirage sont les plus attentifs à ces matières. Ceci étant, même les organismes de presse de qualité et de service public peuvent donner dans le ragot et les insinuations non étayées. Dans la plupart des cas, ils rapportent des controverses nées de révélations faites à l'origine par les tabloïds, mais il leur arrive parfois de prendre les devants. On peut citer, par exemple, les récentes questions posées à Charles Kennedy, chef de file des Libéraux Démocrates, sur ses habitudes en matière de consommation d'alcool dans l'émission d'actualités Newsnight, programme phare de la BBC. Ces questions ont soulevé une pluie de controverses et conduit l'animateur de l'émission à présenter publiquement ses excuses.

$\mathrm{Au}$ sein de la culture journalistique britannique, les questions relatives à la probité publique, d'une part, et aux valeurs personnelles et à la moralité, d'autre part, sont devenues effectivement inséparables. Ce rapprochement a un coût parce que le travail d'investigation sur les acti- 
vités des personnalités politiquement influentes à travers le prisme de la personne peut réellement entraver le comportement démocratique des médias. On peut prendre comme exemple la couverture des organismes quasi-gouvernementaux dans les médias en Grande-Bretagne. Entre 1992 et 1995, on a assisté à une augmentation soudaine de la couverture médiatique de ces «quangos » au Royaume-Uni, qui a suscité et stimulé un vaste débat politique sur leur influence et leur rôle croissants. Mais, à mesure que ce débat médiatique s'intensifiait, il s'est rétréci pour se limiter en définitive à une discussion sur l'abus du népotisme (« du travail pour les enfants ») de la part du gouvernement et sur les actes spécifiques de corruption personnelle et les pratiques douteuses dont les salariés de ces organismes se rendent responsables. La personnalisation progressive de ces questions a détourné involontairement l'attention de ce que beaucoup de commentateurs informés considéraient comme les questions essentielles posées par l'évolution rapide des «quangos", à savoir les carences structurelles dans la responsabilité de ces organismes à l'égard du public et du parlement. Ces arguments concernant ce « déficit démocratique » croissant dans la vie politique britannique ne reposent pas sur la mise en évidence de la corruption des mandataires politiques : leur principal impact concerne la marginalisation croissante de l'électorat dans l'octroi de l'autorité politique'.

Il convient d'ajouter que l'attention croissante des médias à la vie privée des politiques n'a pas nécessairement conduit, dans l'ensemble, à une approche d'investigation et d'interrogation vis-à-vis de l'administration. Parfois, les attaques contre les valeurs et la vie personnelles des politiques constituent une option facile qui évite de se frotter à la culture d'information hautement contrôlée qui entoure, depuis des siècles, l'activité de l'État au Royaume-Uni. Par exemple, les nombreuses études sur l'introduction des nouvelles mesures de liberté de l'information au Royaume-Uni ont révélé que celles-ci sont constamment et manifestement sous-utilisées par les journalistes dans leurs activités ordinaires ${ }^{2}$. Le Royaume-Uni a peut-être des médias acerbes, mais le doute subsiste quant à savoir s'ils sont vraiment des médias d'investigation.

1 P. Dunleavy, S. Weir et G. Subrahmanyam, «Public Response and Constitutional Significance », Parliamentary Affairs, ${ }^{\circ}$ 48, 1995.

2 J. Steele, Public Access to Information. An Evaluation of the Local Government (Access to Information) Act 1985, Policy Studies Institute/Department of the Environment, 1995, paragraphe 9.1. 


\section{Les élections législatives de 2005}

La secrétaire d'État à la culture, Tess Jowell, s'est faite l'écho de nombreux de ses pairs lorsqu'elle s'est plainte de ce que les élections législatives de 2005 étaient réellement constituées de deux campagnes distinctes. Les opposants de la seconde campagne, la moins évidente, n'étaient pas les deux principaux partis rivaux, mais les candidats luttant pour obtenir un soutien et les journalistes cherchant à relater leurs efforts. Mme Jowell a soutenu un avis largement répandu selon lequel il y avait deux guerres distinctes, l'une «aérienne » et l'autre «terrestre », et que cette dernière avait probablement une dimension plus critique pour des élections qui étaient considérées depuis longtemps comme une initiative visant uniquement à encourager les gens à voter, après la forte baisse de la participation observée lors du scrutin précédent. Les politiques et les journalistes ont conjugué leurs efforts pour attirer l'attention du public, et même leurs appuis allaient être attaqués de plusieurs manières. La plus manifeste a été la multitude de sujets antagonistes qui se sont disputés la une des journaux, y compris des événements comme la disparition et les obsèques du Pape et l'élection du nouveau Pape ainsi que le mariage, longtemps attendu, du Prince de Galles et sa compagne, Camilla Parker-Bowles. Outre ces faits, des événements dramatiques comme l'annonce de l'effondrement imminent de la société automobile MG Rover et l'agression brutale, apparemment non provoquée, d'une jeune mère, ont également détourné l'attention des médias des politiques et des élections. Pourtant, la dominance combinée de la culture du vedettariat et du mode de vie, notamment mais pas exclusivement dans les journaux populaires à scandales, a sans doute souligné que les élections et ceux qui s'y affrontaient devraient se battre encore plus pour être vus et entendus sous une lumière favorable. Dans ce cas, il n'est peut-être pas surprenant qu'une des interventions politiques médiatiques les plus efficaces, au cours de la dernière législature, ait été la croisade parrainée par le cuisinier Jamie Oliver contre la mauvaise qualité des aliments servis aux enfants dans les écoles de la nation. Ce type d'interventions populistes et leur côté informel propre aux vedettes a également caractérisé la campagne de 2005.

Depuis au moins 1992 et en certaines occasions auparavant, les élections législatives britanniques ont été précédées d'une "phoney » (élections factices) ou pré-élections lors desquelles les grandes personnalités politiques s'en remettent de plus en plus aux conseillers en image, spécialistes de la communication politique et autres experts de 
la présentation pour promouvoir leur candidature. La fréquence de ces pratiques a conduit de nombreux observateurs à prétendre que la politique est paralysée par une campagne permanente. Pourtant, alors que cet état de fait aurait pu se produire durant la première législature du Parti travailliste, l'invasion de l'Irak a sérieusement gêné les tentatives du gouvernement de gérer son ordre du jour au cours de la seconde législature'. L'Irak a nui à l'image publique de Tony Blair et a remis en question sa crédibilité en la matière et dans toute une série de domaines. Dans leur approche de la campagne de 2005, M. Blair et ses stratèges ont tenté de reprendre l'initiative en lançant la stratégie, dite du « masochisme », centrée sur des apparitions du Premier ministre dans divers formats d'émissions afin de regagner la confiance des électeurs qui l'avaient abandonné. Le Premier ministre a agi de la sorte en sachant que sa fonction et ses qualités médiatiques lui conféraient un net avantage sur ses rivaux, Charles Kennedy et surtout Michael Howard.

L'événement le plus significatif de la pré-campagne concerne les leaders des trois principaux partis qui ont été, à des dates différentes, les invités d'une émission de Channel 5. Bien que ces apparitions ne faisaient pas partie des élections officielles, elles ont été diffusées en février, une période déterminante car des millions de personnes prenaient une décision concernant leur vote ${ }^{2}$. Ce facteur a été sans conteste important pour encourager Tony Blair et ses adversaires à répondre aux questions des intervieweurs dans des éditions spéciales de The Wright Stuff, émission d'actualités qui fait participer le public et est diffusée dans la matinée sur Channel 5, et dans un débat de début de soirée présenté par Kirsty Young. Dans ce dernier format, les leaders ont été soumis à un flot soutenu de questions ardues, notamment parce qu'ils étaient interrogés par une demi-douzaine de membres du public qui leur ont posé, à tour de rôle et pendant plusieurs minutes, des questions sur un sujet qui leur tenait particulièrement à cœur. Par exemple, M. Blair a été poussé dans ses derniers retranchements par une infirmière qui lui a demandé s'il serait prêt à travailler dans un service de santé en effectuant des tâches déplaisantes mais vitales pour un salaire relativement modeste. Le caractère direct de l'intervieweuse et de sa collègue a été mis en exergue par la presse nationale, et les interventions fouillées de certains hommes de l'assistance sur l'Irak, les frais de scolarité et l'enseigne-

1 N. Ornstein and T.Mann, The Permanent Campaign and Its Future. Washington : AEI and Brookings Insititute, 2000.

2 W. Miller et al., How Voters Change, Oxford : Clarendon, 1990 
ment ne sont non plus pas passées inaperçues. Au terme de l'émission, l'impact de ces interviews extrêmement bien réalisées a toutefois été atténué par l'intervention perturbatrice d'une femme qui a interpellé $\mathrm{M}$. Blair en se plaignant du traitement infligé à son fils. Bien qu'elle ait été montée en épingle et exploitée par les Conservateurs, cette affaire a sans doute conforté le message de M. Blair qui s'était dit prêt à écouter les électeurs en colère. Pourtant, comme la plus grande partie du débat de ces élections, les échanges plus fouillés qui ont eu lieu ce jour-là entre électeurs et politiques sur Channel 5 ont été éclipsés par l'annonce d'un événement imprévu mettant en scène un protagoniste charismatique, d'une dimension humaine implacable et/ou assorti de bonnes images.

Le Premier ministre, aidé par un Alastair Campbell adoptant un profil bas - ce qui n'est guère coutumier chez lui - et par David Hill, successeur de Campbell au 10 Downing Street, a profité de sa fonction de différentes manières et à un degré presque sans précédent. Mise à part la stratégie du masochisme, M. Blair a été invité dans une série de programmes parmi les moins formels qu'il courtisait depuis longtemps, mais qui lui ont consacré peu de temps d'antenne avant l'annonce de son élection. Parmi ces programmes, on peut citer un débat avec un public de jeunes invités dans l'émission T4 du dimanche midi sur la chaîne populaire Channel 4. Ce débat, diffusé en janvier, a été précédé d'un film du présentateur June Sarpong qui a fait de l'ombre au Premier ministre. Les commentateurs des médias se sont montrés cyniques quant à la prestation de M. Blair, mais certains téléspectateurs ont été manifestement impressionnés par la sincérité du leader travailliste puisqu'une électrice a admis, plus tard, avoir voté pour le Parti travailliste suite à la «fantastique » performance de son leader ${ }^{1}$. M. Blair a poursuivi ses apparitions télévisuelles, comme invité du Breakfast Show de la chaîne GMTV et de Richard and Judy sur Channel 4, pour se montrer particulièrement attentif aux préoccupations et problèmes des mères d'enfants en âge scolaire et de leur famille ardente au travail, c'est-à-dire les publics cibles déterminés par les sondeurs qui ont joué un rôle significatif dans l'élaboration de l'approche adoptée par le Parti travailliste lors de cette campagne. Le Premier ministre a même accepté d'être l'invité vedette de l'émission-jeu You Say We Pay sur Channel 4, où il a essayé de gagner une somme d'argent pour un téléspectateur.

\footnotetext{
1 The Guardian, 21 avril 2005.
} 
L'incapacité du Premier ministre de répondre à de nombreuses questions dans la partie jeu de l'émission Richard and Judy a révélé d'éventuelles conséquences embarrassantes pour sa stratégie, en particulier qu'il n'était pas en prise directe avec la culture populaire comme il l'avait déclaré1. Néanmoins, sa propension à courtiser les types de publics qui boudent de plus en plus les émissions d'informations, l'a amené à accepter l'invitation lancée par l'émission populaire Saturday Night Takeway sur ITV1, au cours de laquelle il a été interviewé au 10 Downing Street par Ant et Dec, les jeunes animateurs du programme où les jeunes participants se sont moqués de lui. Cette apparition et d'autres encore ont contribué à casser un moule et, il y a une génération d'ici, il aurait été inconcevable pour un Premier ministre en activité de se permettre ce type de comportement par crainte d'une réaction défavorable du public. Cette situation aurait dû se traduire par un surcroît de cynisme de la part des journalistes, mais, en définitive, elle n'a eu qu'une importance marginale par rapport à la possibilité de pouvoir toucher des millions d'électeurs indécis. Les stratèges du Parti travailliste ont également soutenu que ce type d'autopromotion exploitait des atouts qui faisaient défaut à Michael Howard, principal rival de Tony Blair. M. Howard a riposté par une photo aux côtés de sa nombreuse famille, et sa femme a fait un certain nombre d'interventions au cours de la campagne qui s'en est suivi, notamment comme invitée vedette - mais pas très dynamique pour autant - du talk-show de la mi-journée Loose Women sur ITV1. En 2001, l'ex-épouse de Michael Howard, Ffion Hague, avait été curieusement réduite au silence et rarement vue en public, sauf aux côtés de son mari. En revanche, Cherie Booth a joué un rôle plus officiel dans la campagne travailliste en faisant un discours contre la Respect Coalition anti-guerre, même si cette intervention lui a valu moins de réactions du grand public que si elle avait attaqué les conservateurs avec la même conviction. Charles Kennedy et son épouse Sarah ont bénéficié d'une couverture considérablement plus importante suite à la naissance de leur nouveau-né, Donald, durant les prémices de la campagne. L'apparition timide de beaucoup de membres de la famille à cette occasion a donné une image largement plus maîtrisée, en opposition flagrante avec les événements capitaux qui avaient précédé la campagne et l'âpreté du scrutin qui était sur le point de se dérouler.

\footnotetext{
1 Marina Hyde, The Guardian, 29 avril 2005.
} 
En net contraste avec 2001, M. Blair a annoncé la date des élections de manière relativement discrète et s'est immédiatement envolé dans son hélicoptère vers la circonscription la plus « serrée » pour son parti, dans le Dorset, où il a été accueilli par la foule photogénique de supporters invités qui l'a suivi dans la plupart de ses déplacements à travers le pays. Les seuls journalistes qui l'ont accompagné dans ses visites provenaient de la BBC, d'ITN, de Sky et de la Press Association car la présence journalistique a été limitée aux médias d'information considérés comme les plus importants. Par conséquent, l'une des caractéristiques marquées de la campagne a été la manière dont les correspondants exclus ont relaté leurs voyages en hélicoptère, voiture, mobile home, scooter voire side-car à travers la Grande-Bretagne, à la poursuite de M. Blair et d'autres personnalités et électeurs flottants. En agissant de la sorte, les politiques et les médias ont corroboré une opinion de plus en plus populaire, à savoir qu'ils doivent sortir des limites de Westminster pour toucher le public et, plus particulièrement, les abstentionnistes des précédentes élections législatives.

Les stratèges des partis reconnaissent de plus en plus combien il est essentiel d'établir des relations avec les médias locaux pour toucher les électeurs plutôt que de se limiter aux médias nationaux, car les journalistes régionaux sont considérés comme plus intéressés par les sujets importants et plus proches de leurs publics. En outre, le manque de contacts préalables entre les médias locaux et le gouvernement central pouvait contribuer à établir un dialogue plus fructueux, dénué du cynisme, voire de la flagornerie qui caractérisent le lobby de Westminster. Les visites - contrôlées et annoncées un peu à la dernière minute - de différentes personnalités politiques aux sièges régionaux montrent que les rencontres entre électeurs et élus ont été relativement peu nombreuses. Cela n'a pas empêché toutefois quelques altercations entre Michael Howard, John Prescott, Charles Kennedy et divers manifestants, protestataires et journalistes comme Michael Crick de Newsnight sur BBC2 et Nick Robinson d'ITV1. Tony Blair a évité en grande partie ces désagréments grâce à un cordon de sécurité très présent et au fait que son parti a soigneusement préparé les «événements » en travaillant uniquement sur invitation. L'un de ces événements a tout de même été « animé » par la fille d'un activiste travailliste du Yorkshire, qui a attaqué M. Blair sur son bilan, dans ce qui avait été jusque là une visite anodine.

La prépondérance des «pseudo-événements » de relations publiques donnant lieu à des rencontres entre les politiques et leurs plus 
fidèles partisans a eu un impact remarquable sur les aspects qui concernent la couverture de la campagne ${ }^{1}$. Mises à part les protestations des manifestants et des journalistes cyniques, les bulletins d'informations ont été caractérisés par le compte rendu relativement stéréotypé d'une succession de visites et de discours basés sur des "petites phrases ». Par contre, les grands débats entre les leaders politiques diffusés en direct par les radios et télévisions durant les dernières étapes de la campagne, ont permis aux membres du public de se libérer directement de leurs frustrations. L'émission Ask the Leader sur ITV1 a consacré des numéros spéciaux aux chefs de file des principaux partis, ainsi qu'à leurs alliés nationalistes écossais et gallois. La séquence avec Michael Howard a donné lieu à un échange particulièrement tendu sur le rôle central accordé à l'immigration dans le camp conservateur et l'accusation selon laquelle cela contribuait à promouvoir le racisme. L'équivalent de Ask the Leader sur la BBC, l'émission débat Question Time, a présenté un seul programme où sont intervenus les trois principaux leaders politiques à raison d'une tranche d'une demi-heure chacun. Tony Blair a été hué, en revanche Charles Kennedy est celui qui s'est en sorti le mieux. Il a semblé plus à l'aise avec les intervieweurs du public que lors de sa rencontre avec Jeremy Paxman, personnalité redoutée de la $\mathrm{BBC}$, dans la série d'interviews consacrées à chaque candidat au poste de Premier ministre ${ }^{2}$. Quelques commentateurs ont considéré le débat de l'émission Question Time comme une nouveauté (à tort, puisque ce même format avait été utilisé en 1983) et l'ont jugé presque aussi bien que le débat en direct entre les leaders, qui est la formule la plus réclamée. Bien que divers journalistes aient plaidé pour une confrontation télévisée de ce type, il faudra probablement attendre longtemps avant qu'un Premier ministre n'accepte d'être confronté en direct à ses principaux opposants.

Si les radio et télédiffuseurs ont donné aux politiques la possibilité de faire leur autopromotion, ces médias d'information ont également essayé de mettre en avant leurs propres sujets d'actualité. Par exemple, l'émission The Politics Show de la BBC et les autres programmes du

1 D. Boorstin, The Image. London : Weidenfeld \& Nicolson, 1961.

2 Philip Cowley, qui a participé à ce travail, était présent lors du débat en studio et a reconnu que M. Blair avait été désavantagé car il est intervenu en dernier lieu à un moment où la température avait énormément grimpé dans le studio à cause de la lumière des projecteurs et où les passions s'étaient déchaînées parmi les membres du public. 
présentateur Andrew Neil ainsi que Newsnight de la BB2 et son numéro spécial du samedi, ont tenté d'offrir leur propre vision des événements. Dans le même ordre d'idées, la préoccupation concernant l'engagement des électeurs a conduit à la création de Ballot Box Jury sur ITV1 et ses interviews d'électeurs flottants, tandis que la 'Student House' de Newsnight a rempli une fonction similaire, en montrant comment un groupe de jeunes largement indécis s'est forgé une opinion au cours de la campagne. Sky News a fait de même en se concentrant sur les préoccupations du public dans la circonscription très serrée et déterminante de Darwen et Rossendale dans le Lancashire. Des motivations analogues ont servi de base à la couverture régionale assurée par les services locaux d'informations de la BBC et d'ITV et, si ce n'était pas assez, des observateurs des élections ont pu également suivre la campagne $24 \mathrm{~h} / 24$ sur les chaînes d'informations BBC 24, ITV, Sky et CNN, dont certaines ont retransmis en direct les conférences de presse matinales du parti.

L'importance accrue de l'image et le débat suscité par ce phénomène ont aiguisé l'indiscrétion et les interrogations des médias à propos des revendications bien orchestrées des divers partis; Factcheck sur Channel 4, Unspun sur ITV1 et d'autres magazine d'information ont consciemment appliqué une technique popularisée dans les comptes rendus politiques américains consistant à soumettre les déclarations des candidats, ainsi que les revendications et les actions sur lesquelles ils les basent, à l'examen d'experts. Des spécialistes du «langage corporel » sont aussi apparus sur au moins trois des réseaux terrestres pour discourir longuement sur ce que les gestes des politiques pourraient nous révéler sur eux et sur leurs motivations. L'un d'entre eux est apparu dans la série de documentaires Election Unspun sur Channel 4, qui ont également mis le doigt sur des aspects déterminants de certaines matières qui risquaient d'être passées sous silence durant la campagne centrale. Dans cette optique, BBC3 a présenté un film bizarre sur l'histoire des «perturbateurs » (qui veulent troubler les orateurs politiques), et deux de ses stagiaires ont tenté sans succès de décontenancer divers politiques lors d'événements en direct. En revanche, ils ont réussi à exaspérer le service de presse conservateur. Ailleurs, Panorama sur $\mathrm{BBC} 1$ a poursuivi sa série de reportages corrosifs sur les différentes politiques sociales qui, bien que n'étant pas explicitement liés aux élections, étaient tout de même d'une grande pertinence.

Le tableau ci-dessous dresse la liste des dix sujets les plus couverts par les médias d'information nationaux aux cours de la campagne officielle (du 4 avril au 6 mai 2005). Le sujet dominant a porté sur 
le processus des élections, une combinaison hybride comprenant des reportages sur les sondages d'opinion, les stratégies des partis, les initiatives publicitaires et des thèmes connexes. Il ne s'agit en aucun cas d'un phénomène nouveau, mais il convient de noter que l'attention accordée à ce sujet a été plus importante que lors du dernier scrutin, au cours duquel le débat politique a été caractérisé par une critique du « conditionnement de l'image » de la part des médias et de l'opposition. Dans ce cas-ci, un des sujets de préoccupation spécifiques a été l'augmentation évidente de la désaffection de l'électorat et la manière dont elle pourrait être analysée et mieux appréhendée. Le deuxième thème par ordre d'importance concerne les pratiques répréhensibles, un thème apparenté à la corruption, qui est apparu en 1997, et la conduite des politiques (principalement le coup de poing assené par John Prescott à un manifestant) en 2001. Mais ici, l'accent a été mis sur les allégations relatives aux nouvelles règles qui ont favorisé une forte hausse du vote par correspondance. Il est significatif de constater qu'une déclaration extrêmement critique de Richard Mawrey, juge qui a présidé un procès de fraude à Birmingham, mettant en cause les vainqueurs travaillistes des élections locales de 2004, est tombée à point nommé pour intensifier les critiques contre le gouvernement. Le Daily Mail s'est montré particulièrement véhément dans ses attaques contre les ministres et a déclaré qu'un tel degré de vote par correspondance équivalait à une « corruption de la démocratie elle-même».

Sujets à l'agenda : Top 10 des thèmes couverts par les médias nationaux ${ }^{1}$

\begin{tabular}{|c|l|c|}
\hline Thèmes & $\mathbf{2 0 0 1}$ & Pourcentage \\
\hline 1 & Travail & $39 \%$ \\
\hline 2 & Europe & $9 \%$ \\
\hline 3 & Santé & $6 \%$ \\
\hline 4 & Conduite des politiciens & $6 \%$ \\
\hline 5 & Impôts & $6 \%$ \\
\hline 6 & Criminalité & $4 \%$ \\
\hline 7 & Education & $4 \%$ \\
\hline 8 & Services publics & $4 \%$ \\
\hline 9 & Sécurité sociale & $3 \%$ \\
\hline 10 & Autres & $19 \%$ \\
\hline
\end{tabular}

1 Loughborough University Communication Research Centre, The Guardian, $2^{\text {nd }}$ May 2005. 


\begin{tabular}{|c|l|c|}
\hline Thèmes & $\mathbf{2 0 0 5}$ & Pourcentage \\
\hline 1 & Processus électoral & $44 \%$ \\
\hline 2 & Pratiques répréhensibles & $8 \%$ \\
\hline 3 & Irak & $8 \%$ \\
\hline 4 & Immigration/droit d'asile & $7 \%$ \\
\hline 5 & Impôts & $5 \%$ \\
\hline 6 & Santé & $4 \%$ \\
\hline 7 & Criminalité & $4 \%$ \\
\hline 8 & Economie & $4 \%$ \\
\hline 9 & Education & $3 \%$ \\
\hline 10 & Autres & $13 \%$ \\
\hline
\end{tabular}

Alors que la question de la fraude dans les votes par correspondance a semblé favoriser le camp des Conservateurs, le troisième sujet - l'Irak - a renforcé celui des Libéraux Démocrates. Bien que l'invasion n'ait pas constitué un élément majeur dans les étapes initiales de la campagne, elle est devenue un point clé du débat plus tard, avec la fuite puis la publication du mémorandum controversé du procureur général Lord Goldsmith sur la légitimité du soutien de M. Blair à l'action militaire du gouvernement des États-Unis, en l'absence de sanction de l'ONU. Les déclarations de Lord Goldsmith ont été passées à la loupe et, ce qui est plus important, le sujet a sans doute été réexaminé au moment le plus inopportun pour le Parti travailliste. Une fois encore, le Premier ministre a été attaqué sur son intégrité personnelle par Michael Howard, qui l'a traité de «menteur», et Charles Kennedy a encore critiqué le jugement de M. Blair. Alors que l'Irak a dominé en partie la dernière semaine de campagne, le deuxième sujet par ordre d'importance - le droit d'asile et l'immigration - a été au centre du débat durant la quinzaine précédente. L'attention accordée à cette question par les médias avait été soutenue par la manière dont les journaux avaient couvert une affaire remontant à plusieurs années et, plus particulièrement, une série controversée d'articles qui ont fait les gros titres de l'Express, vers la moitié de la législature. Les principales déclarations qui soulignaient que le gouvernement ne parvenait pas à contrôler l'immigration et que la politique d'asile était un véritable chaos, ont repris vigueur du fait que les Conservateurs se sont concentrés sur cette problématique, un des rares sujets où ils devançaient sensiblement les Travaillistes dans les sondages. La condamnation de l'immigrant clandestin Kamel Bourgass pour le meurtre d'un agent de police a donné 
aux détracteurs du gouvernement issus des médias et du monde politique une occasion unique d'aborder le dossier.

L'une des caractéristiques symptomatiques de ces élections est le petit nombre de sujets politiques de fond qui ont été abordés. Même dans les lieux où l'on a abordé des sujets de la vie quotidienne qui présentent un intérêt majeur pour les électeurs, les débats ont eu tendance à se focaliser sur des faits particuliers comme l'apparition en milieu hospitalier du staphylocoque doré résistant à la méthicilline ou le cas d'un patient frustré qui attendait une opération, au détriment d'une discussion plus approfondie sur d'autres aspects importants des prestations de sécurité sociale. De même, le thème de l'Europe a été écarté, tout comme d'autres domaines capitaux de la politique publique, comme le transport, l'Irlande du Nord et le logement.

\section{Conclusion}

Le présent article a identifié de nombreuses tendances négatives concernant la manière dont les journalistes respectent et traitent les élus au Royaume-Uni, aussi bien en règle générale que sur le plan du droit à la vie privée. Cet article ne doit pas être entendu comme une plainte nostalgique des temps anciens où les journalistes savaient rester à leur place et se montraient circonspects dans leurs rapports avec les personnalités politiques, notamment concernant leurs affaires privées. Manifestement, une déférence excessive conduit à sa propre tyrannie ; une dose de causticité et de franche indiscrétion joue un rôle légitime dans le fonctionnement de toute démocratie mature et efficace. Des problèmes surgissent toutefois lorsque le cynisme et le conflit deviennent des caractéristiques définitoires de la relation journaliste-politique, comme semble le montrer la tendance dominante au Royaume-Uni. Considérer tous les politiques comme des êtres égocentriques, fourbes et corrompus est une généralisation excessive et aisée, mais c'est, semble-t-il, l'une des approches adoptées par les journalistes. Chose assez curieuse, ces attitudes peuvent souvent entraver plutôt que valoriser le rôle de « chien de garde » joué par les médias : elles fixent l'attention sur les sphères personnelles plutôt qu'officielles des activités des politiques et présentent une opinion excessivement individualisée de l'exercice du pouvoir politique. Attribuer un échec politique persistant uniquement à la vénalité et à l'incompétence des individus peut très aisément nous empêcher de déceler les déficiences du système démocratique britannique. 
Cela pose la question de savoir qui est fondamentalement coupable de cette situation. Les politiques récoltent-ils la tempête qu'ils ont semée en raison de leur propension à exploiter leur vie privée et à s'approprier les conventions de la culture du vedettariat lorsque cela les arrange ? Ou sont-ils tyrannisés par une logique qui n'est pas la leur, obligés de jouer le jeu de la présentation comme le souhaitent les médias s'ils veulent avoir une chance de communiquer avec leur électorat, ce qui les expose dangereusement à l'indiscrétion des médias ? Aborder cette question en termes de cause et d'effet, et chercher ainsi à attribuer la faute originelle soit aux politiques soit aux journalistes, est une approche inappropriée car elle sous-estime la complexité des processus en présence. Comme cela est indiqué dans cet article, il existe une dynamique concurrentielle évidente au sein des systèmes politiques et entre eux, et la combinaison de ces facteurs alimente l'indiscrétion et le cynisme des médias. Par exemple, la promotion croissante des personnalités par les partis politiques est tout autant motivée par l'intensification de la concurrence entre les partis dans une période de valeurs politiques moins clairement délimitées que par la nécessité d'attirer et de contrôler l'attention des médias. De même, le fait que les journalistes privilégient leurs propres valeurs d'information dans leurs reportages sur les politiques est tout aussi directement lié aux pressions du marché et à la nécessité de conserver leur part d'audience qu'à la nécessité de résister à la coercition ou la cooptation des spécialistes du conditionnement de l'image au sein des partis. 